\section{Branching regulation}

Plant hormone strigolactones regulate branching by maintaining the dormant state of axillary buds. A receptor for strigolactones remained unknown, though previous work had implicated a handful of genes in the response to these hor-

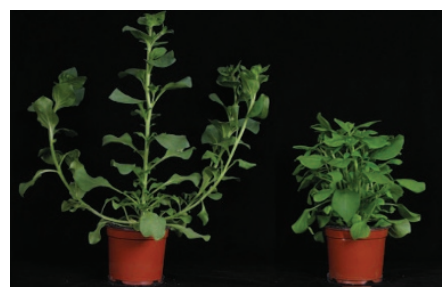
mones. Now work from Snowden and colleagues indicates that the product of one such gene, DAD2 in petunia (D14 in Oryza sativa and Arabidopsis thaliana), is a receptor for strigolactones. The authors cloned the DAD2 gene and solved the crystal structure of the protein, revealing an $\alpha / \beta$ hydrolase fold with a large cavity and a canonical catalytic triad. The cavity is L-shaped and strongly hydrophobic, and it could accommodate strigolactones. In fact, the thermal stability of DAD2 is reduced in the presence of the synthetic strigolactone GR24, indicating protein conformational changes. In addition, when GR24 was incubated with DAD2, the hormone was cleaved into products that lacked inhibitory activity on bud growth. The authors then queried DAD2's interactions with other factors involved in strigolactone signaling. They found that DAD2 interacts with F-box protein PhMAX2A but only in the presence of GR24 and in a dose-dependent manner. A catalytically inactive DAD2 mutant could not complement the dad2 mutation; it was also unable to interact with PhMAX2A in the presence of GR24 or added cleavage products. The authors propose that structural changes in DAD2 that take place after strigolactone binding, perhaps due to the formation of a reaction intermediate, are required for DAD2 to interact with PhMAX2A and hence for signal transduction. This hypothesis is consistent with previous observations that strigolactone analogs that are resistant to cleavage are also inactive. (Curr. Biol. published online 6 September 2012, doi:10.1016/j.cub.2012.08.007)

\section{Death control}

Programmed cell death can be initiated by intrinsic or extrinsic pathways. Extrinsic pathways involve the activation of death receptors (DRs), which triggers the formation of death-induced signaling complexes (DISCs) consisting of caspase- 8 and fas-associated protein with death domain (FADD). DISC formation activates caspase-8, leading to apoptosis. Under certain conditions, caspase- 8 and FADD have also been shown to suppress necroptosis. DR downstream signaling is regulated by various posttranslational modifications, but FADD is only known to undergo phosphorylation, and this modification is not involved in the induction of apoptosis. Lee et al. have now found a role for MKRN1, an E3 ubiquitin ligase that targets $\mathrm{p} 53$ and p21, in FADD regulation. Working in HeLa cells, the authors observed that MKRN1 knockdown induced p53-independent cell death and that addition of a pan-caspase inhibitor reversed those effects, suggesting that MRKN1 is involved in caspase-dependent apoptosis. Further experiments revealed faster DISC formation as well as increased FADD levels upon MKRN1 depletion. In addition, MRKN1 directly ubiquitinates FADD, leading to its proteasomal degradation and negatively regulating DR-mediated apoptosis. Interestingly, MRKN1 knockdown also increased formation of the RIP1-RIP3-FADD necrosome complex, but additional depletion of FADD led to even more RIP1-RIP3 complex formation and more efficient necroptoptic cell death in a mouse fibroblast cell line. These data support a negative role for FADD

Written by Inês Chen, Stéphane Larochelle, Michelle Montoya \& Beth Moorefield in necroptosis and implicate MKRN1 in an uncharacterized role in this pathway. Overall, the findings suggest that MKRN1 prevents killing of normal cells and may also limit cell death in the presence of a DR signal. (Nat. Commun. 3, 978, 2012)

$M M$

\section{Pulling recombination apart}

During meiosis, homologous chromosomes must recombine to establish the physical connections required for their proper segregation. Recombination is initiated by resection of double-strand break (DSB) ends to generate single-stranded DNA tails that associate with the Rad51 and Dmc1 recombinases. The resultant nucleoprotein filaments catalyze double-stranded DNA invasion and strand exchange that links homologous pairs to form joint molecules (JMs). Although Rad51 and meiosis-specific Dmcl co-localize to nuclear foci, and both can promote strand exchange in vitro, it is not clear that they have equivalent roles in meiotic recombination. Now, Bishop and colleagues have created a Rad51 mutation that uncouples filament assembly from JM formation to dissect the functional contributions of each recombinase during meiosis in yeast. They show that specifically abolishing the JM-forming activity of Rad51 has no effect on recombination between homologs during meiosis, whereas the analogous mutation in Dmc1 produces severe recombination defects. However, Rad51 JM activity is essential for mitotic DNA repair. Although JM formation by Dmc1 is sufficient to direct normal interhomolog recombination during meiosis, this activity is regulated by Rad51. The authors show that Rad51 functions with the known Dmc1 cofactor complex Mei5-Sae3 to stimulate formation of recombination intermediates in vitro and that Rad51 JM activity is dispensable for this stimulation. Thus, Rad51 functions indirectly in meiotic recombination by stimulating Dmcl activity and functions directly in mitotic recombination by catalyzing strand exchange. (Science 337, 1222-1225, 2012)

$B M$

\section{Bent out of shape}

The bending of cellular membranes is central to the generation of most organelles and dynamic cellular structures such as endocytic pits. A favored model for protein-induced membrane bending is the insertion of amphipathic helices into one leaflet of the lipid bilayer to create membrane-deforming wedges. However, continuum models and molecular dynamics simulations predict that to induce sufficient bending and produce endocytic structures, epsin 1-an amphipathic helixcontaining protein involved in clathrin-mediated endocytosiswould have to cover $>100 \%$ of the membrane. Stachowiak, Schmid, Fletcher, Hayden and colleagues set out to test the hypothesis that epsin 1 bends membranes by helix insertion, but instead demonstrate that protein crowding can drive bending in the absence of helix insertion. The authors removed the epsin 1 amphipathic helix and added a $\mathrm{His}_{6}$ tag to the ENTH domain of epsin 1 . They then used vesicles containing modified lipids that can bind histidine tags. At similar surface density, membrane curvature is induced by epsin 1's ENTH domain, whether or not a helix is present. The same observations were made with a similarly membrane-targeted GFP, suggesting that protein density per se is capable of inducing bending. It is not yet clear how or even if the ability of protein crowding to bend membranes is at play in vivo. However, the results suggest the interesting possibility that the directionality of membrane curvature could be influenced by the relative density of protein on either side of the membrane. (Nat. Cell Biol. 14, 944-949, 2012) $S L$ 